\title{
Amplifying the Macromolecular Crowding Effect Using Nanoparticles
}

\author{
Ahmed Zaki, Neeshma Dave, and Juewen Liu* \\ Department of Chemistry, Waterloo Institute for Nanotechnology, University of Waterloo, Waterloo, Ontario, N2L 3G1, Canada
}

liujw@uwaterloo.ca

ABSTRACT: The melting temperature $\left(T_{m}\right)$ of DNA is affected not only by salt but also by the presence of high molecular weight (MW) solutes, such as polyethylene glycol (PEG), acting as a crowding agent. For short DNAs in a solution of low MW PEGs, however, the change of excluded volume upon melting is very small, leading to no increase in $T_{m}$. We demonstrate herein that by attaching 12-mer DNAs to gold nanoparticles, the excluded volume change was significantly increased upon melting, leading to increased $T_{m}$ even with PEG 200. Larger AuNPs, higher MW PEGs and higher PEG concentrations show even larger effects in stabilizing the DNA. This study reveals a unique and fundamental feature at nanoscale due to geometric effects. It also suggests that weak interactions can be stabilized by a combination of polyvalent binding and the enhanced macromolecular crowding effect using nanoparticles.

Macromolecules such as proteins, RNA, and DNA occupy 20$40 \%$ of a live cell's volume. ${ }^{1}$ Due to their mutually impenetrable nature, the excluded volume occupied by each macromolecule is larger than its geometric volume, making the addition of new polymers into this crowded system thermodynamically disfavored. This fundamental biophysical effect has a strong influence on many biochemical reactions including protein folding, oligomerization, and DNA hybridization and melting. ${ }^{2}$ Much attention has been given to the process of DNA melting, as this reaction plays an important role in biology and biotechnology. ${ }^{3-8}$ At the same time, DNA length and sequence can be easily varied to allow systematic studies.

Upon melting, the excluded volume of a DNA is increased. Therefore, melting is usually disfavored in macromolecularly crowded environments to give rise to more stable DNA duplex and increased melting temperature $\left(T_{m}\right)$. Polyethylene glycol (PEG) is the most commonly used crowding agent. PEG has been shown to both stabilize and destabilize DNA, depending on PEG molecular weight (MW), DNA length and salt concentration. For example, with 8 to 17 base pair (bp) DNAs in the presence of $1 \mathrm{M}$ $\mathrm{NaCl}$, only destabilization (reduced $T_{m}$ ) was observed even with $20 \%$ PEG $8000 .^{7}$ By reducing the salt to $100 \mathrm{mM}$, stabilization of a 20 bp DNA was observed with $15 \%$ PEG $6000 .{ }^{5}$ On the other hand, with $\sim 200 \mathrm{bp}$ DNAs, an increase in $T_{m}$ was achieved when the PEG MW was greater than $400-1000 .{ }^{4}$ This is because under otherwise identical conditions, longer DNAs produce larger excluded volume increase upon melting. The fact that decreased $T_{m}$ was also sometimes observed suggests that PEGs play a dual role on DNA stability. In addition to the excluded volume effect for stabilization, PEGs also reduce the water activity and solvate DNA bases leading to decreased $T_{m} \cdot{ }^{4,5,7}$ In this paper, macromolecular crowding (MC) describes solely the excluded volume effect while the chemical interaction between PEG and DNA is called the destabilizing chemical effect. Efforts to dissect these two effects have been recently carried out. In general, small PEGs tend to have a strong destabilizing chemical effect and weak MC effect, leading to an overall reduced $T_{m}{ }^{8}$
Based on the understanding of excluded volume, we consider that attaching short DNAs to a larger object, such as a gold nanoparticle (AuNP), might augment the excluded volume change upon DNA melting and thus amplify the MC effect. This hypothesis is supported by a simple geometric calculation that will be discussed later. Since its first report in $1996,{ }^{9,10}$ DNA-linked AuNPs are probably one of the most well-studied systems in bionanotechnology. ${ }^{11,12}$ This system has many unique properties including a blue-to-red color change upon melting and a sharp melting transition. The melting of such DNA-linked AuNPs in high MW PEGs (MW > 8000) with $300 \mathrm{mM} \mathrm{NaCl}$ has been previously reported where DNA melting was qualitatively similar in the presence or absence of AuNPs. ${ }^{13}$ We consider that with such high MW PEGs and salt, even free DNAs were effectively stabilized and the role of AuNPs might have been masked. ${ }^{8}$ Herein, we focus on low MW PEGs in low salt conditions. We report for the first time that the $T_{m}$ of AuNP-attached DNA was increased in the presence of even PEG 200, while free DNAs still showed reduced $T_{m}$. The $T_{m}$ increase positively correlated with AuNP size, supporting that AuNPs can amplify the MC effect.

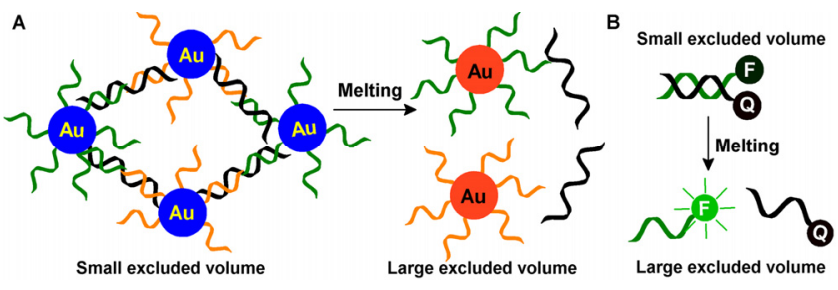

Figure 1. Schematic presentation of the melting of DNA-linked AuNPs (A) and the melting of free DNA based on fluorescence (B). The increase of excluded volume upon melting is much larger in (A) than in (B). The crowding agent (PEG) is not drawn.

We respectively functionalized $13 \mathrm{~nm}$ AuNPs with two kinds of thiol-modified 12-mer DNA and assembled them using a 24mer linker DNA. The temperature of the sample was gradually increased to induce DNA melting and AuNP disassembly as shown in Figure 1A. ${ }^{11}$ Since the melting of either 12-mer DNA can induce AuNP disassembly, a 12-mer DNA duplex in the absence of AuNPs was also prepared as shown in Figure 1B. To achieve high precision and high throughput in $T_{m}$ measurement, these free DNAs were labeled with a fluorophore and quencher respectively and a real-time PCR thermocycler was used to obtain melting curves. This fluorescence method was previously reported, ${ }^{14}$ and a comparison to the tradition method based on the UV hyperchromic effect has been recently carried out. ${ }^{15}$ Based on this report, our fluorescent DNA should give precise and accurate $T_{m}$ results.

The melting curves of the AuNP-free fluorescent DNA in water, $5 \%(\mathrm{w} / \mathrm{w})$ glycerol, ethylene glycol (EG), or PEGs of different MWs are shown in Figure 2A. All the solutions also contained 50 $\mathrm{mM} \mathrm{NaCl}, 10 \mathrm{mM}$ HEPES, pH 7.6. The fluorescence intensity gradually increased with increasing temperature, indicating DNA melting. The sample in water was measured 12 times and the coefficient of variation was only $0.4 \%$ (Figure $2 \mathrm{C}$, red square), 
supporting the outstanding reproducibility of fluorescence measurement. Glycerol, EG, as well as PEG 200 destabilized the DNA, while PEG 2000 and above stabilized it. $T_{m}$ as a function of PEG $\mathrm{MW}$ is shown in Figure 2C. Compared to the sample in water (red square), the $T_{m}$ decreased $\sim 1.2^{\circ} \mathrm{C}$ in PEG 200. PEG 2000, 8000, and 20000 stabilized the duplex but only for $\sim 0.9^{\circ} \mathrm{C}$, which was highlighted by the dashed lines in Figure $2 \mathrm{C}$. This result is consistent with literature reports that the stabilization effect was very small for short DNAs.

Record and co-workers recently reported that small PEGs had stronger destabilizing chemical effects than the excluded volume interaction, resulting in reduced $T_{m} .{ }^{8}$ On the other hand, high MW PEGs had strong excluded volume stabilization while the destabilizing chemical interaction was attenuated since a large fraction of the PEG chains were shielded. The transition to an overall stabilization occurred at PEG 400, and PEGs larger than 2000 did not show further stabilization in the presence of the same PEG monomer unit concentration (i.e. w/w concentration). Our observations in Figure $2 \mathrm{C}$ are in good agreement with this report and thus can be explained by their model.
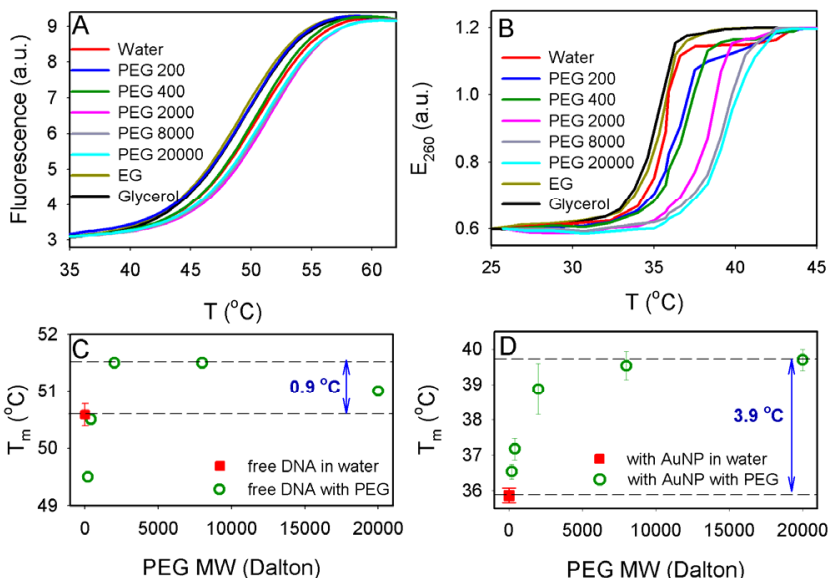

Figure 2. Melting curves of the free DNA (A) and the DNA-linked 13 $\mathrm{nm}$ AuNPs (B) in $5 \%(\mathrm{w} / \mathrm{w})$ solutes. The melting buffer contained 50 $\mathrm{mM} \mathrm{NaCl}, 10 \mathrm{mM}$ HEPES, $\mathrm{pH}$ 7.6. $T_{m}$ as a function of PEG MW for the free DNA (C) and the DNA-linked $13 \mathrm{~nm}$ AuNPs (D). The samples in the absence of any PEG are shown in red. The two dashed lines define the maximal stabilization by $5 \%$ PEG. The error bars represent the standard deviations of independent measurements.

Next the melting curves of DNA-linked $13 \mathrm{~nm}$ AuNPs were measured. Representative TEM micrographs and UV-Vis spectra of these AuNPs are presented in Supporting Information. As shown in Figure 2B, the AuNP extinction at $260 \mathrm{~nm}$ was monitored as a function of temperature. Melting with AuNPs occurred in a much narrower temperature range, which is characteristic of such polyvalent DNA binding systems undergoing cooperative melting transitions. ${ }^{16}$ Interestingly, all of the PEGs induced stabilization in the presence of AuNPs, even for PEG 200; while small molecule solutes (glycerol and EG) still showed reduced $T_{m}$, suggesting the important role of the MC effect acting on the AuNPs. The excluded volume interaction with AuNPs must be so large that it over-compensated the destabilizing chemical effect of PEG 200 on the DNA. This is the first time that an increase in duplex DNA $T_{m}$ was observed for such small PEGs and short DNA, ${ }^{5,17}$ highlighting the crucial role of AuNPs. With higher MW PEGs, a $T_{m}$ increase up to $3.9{ }^{\circ} \mathrm{C}$ was achieved (Figure 2D), which was more than four fold of that for the free DNA. In this regard, the MC effect was amplified by the presence of AuNPs.
So far, we have demonstrated an amplified stabilization effect by confining DNA at the surface of $13 \mathrm{~nm}$ AuNPs. Next, we aim to test the effect of AuNP size. For this purpose, we prepared 5, 13, 20, and $30 \mathrm{~nm}$ AuNPs functionalized with the same DNAs. Their melting curves were measured in 10\% PEG 200. As shown in Figure 3A, stabilization was observed even with $5 \mathrm{~nm}$ AuNPs, although the $T_{m}$ shift was only $\sim 0.9{ }^{\circ} \mathrm{C}$. Very large $T_{m}$ shifts exceeding $3{ }^{\circ} \mathrm{C}$ were achieved with 20 and $30 \mathrm{~nm}$ AuNPs (Figure $3 \mathrm{~B}, \mathrm{C})$. The $T_{m}$ increase was plotted as a function of AuNP size in Figure 3D (red dots); larger AuNPs produced a higher increase in $T_{m}$.

With a high density of polyanionic DNA, these AuNPs are known to enhance the local cation concentration, which stabilizes duplex DNA. ${ }^{14,18}$ The effect of salt on DNA melting, ${ }^{19}$ and especially in a crowded environment has been carefully studied. ${ }^{4-6}$ To understand whether the presence of AuNPs can affect the association of salt with DNA in such a crowded environment, we measured the $T_{m}$ of AuNPs as a function of $\mathrm{NaCl}$ concentration. As shown in Figure 3E, a linear relationship was obtained both in the presence and absence of $10 \%$ PEG 200, which is typical for DNA melting. The slope of these curves is proportional to the number of sodium ions bound to DNA upon duplex formation or released upon melting. ${ }^{4-6}$ A smaller slope was observed in the presence of PEG, suggesting that PEG reduced the number of $\mathrm{Na}^{+}$associated with DNA upon forming AuNP aggregates and this is similar to the behavior of free DNA. ${ }^{4-6}$ Figure $3 \mathrm{E}$ also showed that the $T_{m}$ shift was larger in lower salt buffers. For example, with $15 \mathrm{mM}$ $\mathrm{NaCl}, 10 \%$ PEG 200 was able to increase $T_{m}$ by $\sim 6^{\circ} \mathrm{C}$ for the 13 $\mathrm{nm}$ AuNPs. This property has also been reported for free DNA that the crowding effect was more pronounced for weak interactions. $^{4-6}$ Therefore, the presence of AuNPs did not change the interaction between salt and DNA.
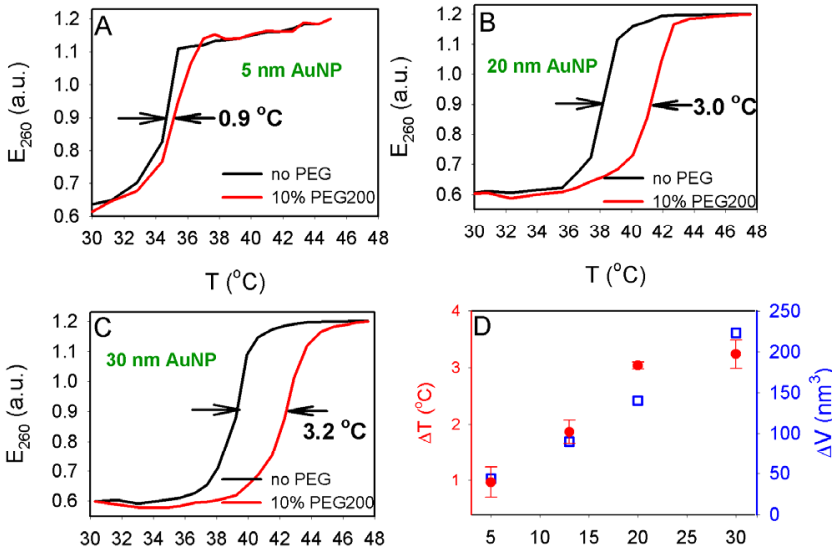

$\mathrm{T}\left({ }^{\circ} \mathrm{C}\right)$
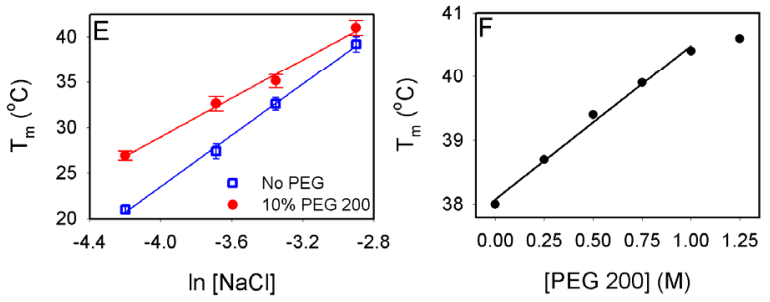

Figure 3. The melting curves of DNA-linked AuNPs (A) $5 \mathrm{~nm}$, (B) $20 \mathrm{~nm}$, and (C) $30 \mathrm{~nm}$ in the presence and absence of 10\% PEG 200. (D) The increase of $T_{m}$ (red dots, the left axis) and the calculated increase of the excluded volume (blue dots, the right axis) as a function of AuNP size. (E) The effect of $\mathrm{NaCl}$ concentration on $T_{m}$. The $x$-axis is the natural logarithm of $[\mathrm{NaCl}]$ in molar. (F) The effect of PEG concentration on $T_{m}$ of $13 \mathrm{~nm}$ AuNPs. $1 \mathrm{M}$ of PEG 200 is $\sim 20 \%$ $(\mathrm{w} / \mathrm{w})$. 
We rationalize our observations based on the model shown in Figure 4A. First, if only two AuNPs are linked by DNA to form a dimer and PEGs cannot penetrate through the DNA layer, the excluded volume of this dimer is the volume encased by the blue solid line. The thickness of this line is determined by the size of PEG, which is $\sim 0.4 \mathrm{~nm}$ for PEG 200 (i.e. the hydrodynamic radius). ${ }^{20}$ Here, PEG is treated as a hard sphere (e.g. the green spheres in the figure), and its center is excluded from the blue line. Therefore, the excluded volume becomes much larger with high MW PEGs. In the absence of PEG, the excluded volume is the same as the geometric volume and there is no crowding effect. By treating PEGs as hard spheres we assumed that they were in the dilute regime. This assumption is supported by the fact that the onset of chain overlap occurs at $\sim 33 \%$ for PEG $200,{ }^{21}$ which is much higher than the $5-10 \%$ concentration range used in this work.

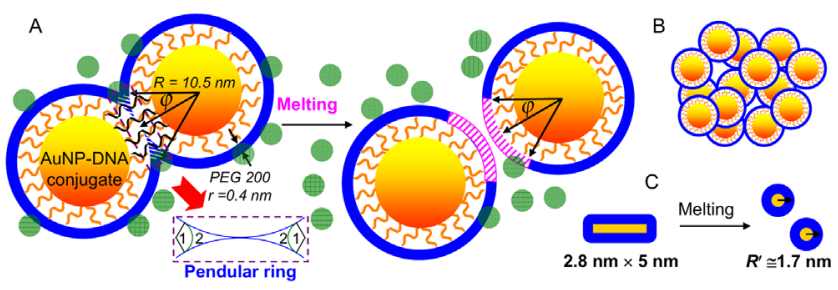

Figure 4. (A) Schematics of excluded volume change after melting of a DNA-linked AuNP dimer (13 nm AuNP in PEG 200). The blue solid lines encase the excluded volumes. The regions of interest for calculating excluded volume increase form a pendular ring (shaded) and defined by the $\varphi$ angle. This pendular ring prior to melting is divided into parts 1 and 2 for volume calculation (see Supporting Information). After melting, the same regions are shaded in pink. (B) There are multiple neighbors for each AuNP, producing an even larger increase in the excluded volume upon melting. (C) Schematics of excluded volume change for a 12-mer duplex upon melting. The dimensions are estimated based on zero excluded volume change. Drawings are not to scale.

After melting, the excluded volume increases. This increase originates only from the pendular ring between the two AuNPs, as the rest is not affected by melting. The volume of this pendular ring before melting is given by $V_{p}=F(R, \varphi)$, where $R$ is the radius of the DNA-functionalized AuNP and $\varphi$ is the angle related to the PEG and AuNP size. $\varphi$ defines the boundary of the pendular ring (i.e. PEG is excluded from the volume within the $\varphi$ angle). For 13 $\mathrm{nm}$ AuNPs in the presence of PEG 200, this pendular volume is calculated to be $10.7 \mathrm{~nm}^{3}$. After melting, the new excluded volume from this region is calculated to be $101 \mathrm{~nm}^{3}$, giving an increase of $90.3 \mathrm{~nm}^{3}$. If the AuNP size is increased to $30 \mathrm{~nm}$, the increase of the excluded volume reaches $223 \mathrm{~nm}^{3}$, which is consistent with our observation that the $T_{m}$ shifts more for larger AuNPs. The calculated excluded volume change is plotted as a function of AuNP size in Figure 3D (blue squares) and a roughly linear relationship is observed. The change of $T_{m}$, however, is not linear (red dots). The model presented here is an idealized geometric model with a number of assumptions and simplifications. For example, it does not consider the DNA conformational change upon melting, the number of DNA linkages between AuNPs, or the excluded volume of the linker DNA. Building a more precise model will be a subject of future studies. The detailed geometric deduction of the current model is included in Supporting Information.

So far, we only considered the melting of two AuNPs. The excluded volume change is further amplified if we consider that each AuNP aggregate contains hundreds to thousands of AuNPs and each AuNP can, maximally, be in contact with twelve others in a close packed form. Therefore, on a per particle basis, the above calculated excluded volume increase should be multiplied by a factor up to six. ${ }^{22,23}$ It is known that under our experimental conditions the packing of AuNPs was likely to be non-crystalline and fractal (Figure 4B), ${ }^{24}$ giving a factor smaller than six.

In the above discussion, we fixed the PEG MW to be 200 and varied the AuNP size. In the following, the AuNP size is fixed and PEG MW and concentration are varied. For this purpose, the following equation describes the change of melting temperature $\Delta T_{m}$ due to the excluded volume change $\Delta V_{e x}$ upon DNA melting. 4

$\Delta T_{m}=\frac{R T_{m}^{0^{2}}}{\Delta H} \Delta V_{e x} C_{p}$

where $R$ is the gas constant, $\Delta H$ is the enthalpy of DNA melting, $T_{m}{ }^{0}$ is the $T_{m}$ in the absence of PEG, and $C_{p}$ is the molar concentration of PEG. Equation (1) predicts a linear relationship between $\Delta T_{m}$ and $C_{p}$. We performed PEG 200 concentration-dependent $T_{m}$ measurements using $13 \mathrm{~nm}$ AuNPs. As shown in Figure 3F, such a linear relationship was indeed observed, further confirming that the MC effect was taking place even with AuNPs. The original melting traces and also the melting of $30 \mathrm{~nm}$ AuNPs are presented in Supporting Information.

Usually $\Delta H$ does not change much in the presence of PEG. ${ }^{5,8,25}$ Therefore for PEGs of different MW, $\Delta T_{m}$ is mainly determined by $\Delta V_{e x}$ and $C_{p}$. According to our geometric model, higher MW PEGs should give rise to larger $\Delta V_{e x}$. For example, we estimated that $\Delta V_{e x}$ for a $13 \mathrm{~nm}$ AuNP dimer with PEG 2000 and 8000 to be 492 and $1917 \mathrm{~nm}^{3}$, respectively. With a concentration of $5 \%(w / w)$, the molar concentration of PEG 8000 was only a quarter of that of PEG 2000. The fact that they had a similar $\Delta T_{m}$ in Figure 2D also supported our geometric model.

Even with AuNPs, the destabilizing chemical effect can still act on DNA. This can be concluded from the reduced $T_{m}$ in the presence of glycerol and EG. Taking advantage that free DNA melting is a fully reversible and two-state process, Record and coworkers were able to accurately measure the equilibrium constant and subsequently determine the contribution of the destabilizing and stabilizing effects. ${ }^{8}$ With AuNPs, however, a two-state model cannot be established and the reported method cannot be directly applied. Nevertheless, we can still conclude that for DNA-linked AuNPs, the excluded volume interaction played a major role in the presence of PEG, since an overall increase in $T_{m}$ was observed for all the PEGs.

In summary, there are several important conclusions and implications from this study. First, the effect of PEG on DNA melting is described as the MC effect by biochemists and biophysicists. In the field of colloidal particle aggregation, ${ }^{26}$ the same fundamental interaction is known as the depletion force. We consider that the amplified MC effect observed in this work can also be explained using the attractive depletion force. DNAfunctionalized AuNPs thus offer a highly tunable experimental system to bridge these two communities. Second, the change of physical property as a function of nanoparticle size is a hallmark of nano-science. Most of the previous work focused only on properties such as light absorption, emission, phase transition, and melting. ${ }^{11,27}$ We show here that the simple geometric interaction can also have a strong nanoscale effect. Third, we may not even need to have large biomolecules to observe the MC effect; even small molecules can be turned into macromolecules as long as they are attached to nanoparticles. For example, the 12-mer DNA is, by itself, considered a small molecule in terms of the crowding effect. It did not show a significant change in the excluded volume upon melting, but the crowding effect was observed when functionalized to AuNPs. Fourth, DNA-functionalized nanomate- 
rials have already been used as analytical probes and drug carriers. $^{28}$ This study indicated that the property of DNA is affected by AuNPs. Therefore, the related MC effect needs to be taken into consideration, especially for cellular applications. Finally, this study provides a useful means to stabilize weak interactions. By attaching a large number of molecules to nanoparticles in a crowded environment, weak interactions may still lead to stable interactions because of both polyvalent binding and the enhanced $\mathrm{MC}$ effect.

ACKNOWLEDGMENT. This work is supported by the University of Waterloo, Canada Foundation for Innovation, Ontario Ministry of Research and Innovation, and the Natural Sciences and Engineering Research Council (NSERC) of Canada. We thank Dr. Jean Duhamel and the anonymous reviewers for insightful comments and suggestions.

Supporting Information Available: Materials and methods, geometric calculations, TEM micrographs, UV-Vis spectra. This material is available free of charge via the Internet at http://pubs.acs.org.

\section{REFERENCES}

(1) Zimmerman, S. B.; Minton, A. P. Ann. Rev. Biophys. Biomol. 1993, 22, 27-65.

(2) (a) Minton, A. P. J. Biol. Chem. 2001, 276, 10577-10580. (b) Ellis, R. J. Trends Biochem. Sci. 2001, 26, 597-604. (c) Zhou,

H.-X.; Rivas, G.; Minton, A. P. Ann. Rev. Biophys. 2008, 37, 375-397. (d) Miyoshi, D.; Sugimoto, N. Biochimie 2008, 90, 1040-1051.

(3) (a) Woolley, P.; Wills, P. R. Biophys. Chem. 1985, 22, 89-94. (b) Miyoshi, D.; Matsumura, S.; Nakano, S.-i.; Sugimoto, N. $J$. Am. Chem. Soc. 2003, 126, 165-169. (c) Miyoshi, D.; Karimata, H.; Sugimoto, N. J. Am. Chem. Soc. 2006, 128, $7957-$ 7963. (d) Smith, B. D.; Liu, J. J. Am. Chem. Soc. 2010, 132, 6300-6301.

(4) Spink, C. H.; Chaires, J. B. Biochemistry 1999, 38, 496-508.

(5) Goobes, R.; Kahana, N.; Cohen, O.; Minsky, A. Biochemistry 2003, 42, 2431-2440.

(6) Karimata, H.; Nakano, S.; Sugimoto, N. Bull. Chem. Soc. Jpn. 2007, 80, 1987-1994.

(7) Nakano, S.-i.; Karimata, H.; Ohmichi, T.; Kawakami, J.; Sugimoto, N. J. Am. Chem. Soc. 2004, 126, 14330-14331.

(8) Knowles, D. B.; LaCroix, A. S.; Deines, N. F.; Shkel, I.; Record, M. T. Proc. Natl. Acad. Sci. USA 2011, 108, 1269912704.

(9) Mirkin, C. A.; Letsinger, R. L.; Mucic, R. C.; Storhoff, J. J. Nature 1996, 382, 607-609.

(10) Alivisatos, A. P.; Johnsson, K. P.; Peng, X.; Wilson, T. E.; Loweth, C. J.; Bruchez, M. P., Jr; Schultz, P. G. Nature 1996, 382, 609-611.
(11) Elghanian, R.; Storhoff, J. J.; Mucic, R. C.; Letsinger, R. L.; Mirkin, C. A. Science 1997, 277, 1078-1080.

(12) (a) Storhoff, J. J.; Lazarides, A. A.; Mucic, R. C.; Mirkin, C. A.; Letsinger, R. L.; Schatz, G. C. J. Am. Chem. Soc. 2000, 122, 4640-4650. (b) Jin, R.; Wu, G.; Li, Z.; Mirkin, C. A.; Schatz, G. C. J. Am. Chem. Soc. 2003, 125, 1643-1654. (c) Harris, N. C.; Kiang, C. H. J. Phys. Chem. B 2006, 110, 1639316396. (d) Smith, B. D.; Dave, N.; Huang, P.-J. J.; Liu, J. J. Phys. Chem. C 2011, 115, 7851-7857.

(13) Goodrich, G. P.; Helfrich, M. R.; Overberg, J. J.; Keating, C. D. Langmuir 2004, 20, 10246-10251.

(14) Lytton-Jean, A. K. R.; Mirkin, C. A. J. Am. Chem. Soc. 2005 127, 12754-12755.

(15) You, Y.; Tataurov, A. V.; Owczarzy, R. Biopolymers 2011, 95, 472-486.

(16) Jin, R.; Wu, G.; Li, Z.; Mirkin, C. A.; Schatz, G. C. J. Am. Chem. Soc. 2003, 125, 1643-1654.

(17) Pramanik, S.; Nakamura, K.; Usui, K.; Nakano, S.-i.; Saxena, S.; Matsui, J.; Miyoshi, D.; Sugimoto, N. Chem. Comm. 2011 47, 2790-2792.

(18) Zwanikken, J. W.; Guo, P.; Mirkin, C. A.; Olvera de la Cruz, M. J. Phys. Chem. C 2011, 115, 16368-16373.

(19) Anderson, C. F.; Record, M. T. Ann. Rev. Phys. Chem. 1995, 46, 657-700.

(20) Kuga, S. J. Chromatogr A 1981, 206, 449-461.

(21) Brandrup, J.; Immergut, E. H.; Grulke, E. A.; Abe, A.; Bloch, D. R.; John Wiley \& Sons: 2005.

(22) Park, S. Y.; Lytton-Jean, A. K. R.; Lee, B.; Weigand, S.; Schatz, G. C.; Mirkin, C. A. Nature 2008, 451, 553-556.

(23) Nykypanchuk, D.; Maye, M. M.; van der Lelie, D.; Gang, O. Nature 2008, 451, 549-552.

(24) Park, S. Y.; Lee, J.-S.; Georganopoulou, D.; Mirkin, C. A.; Schatz, G. C. J. Phys. Chem. B 2006, 110, 12673-12681.

(25) Spink, C. H.; Garbett, N.; Chaires, J. B. Biophys. Chem. 2007, 126, 176-185.

(26) Park, K.; Koerner, H.; Vaia, R. A. Nano Lett. 2010, 10, 1433 1439.

(27) Alivisatos, A. P. Science 1996, 271, 933-937.

(28) (a) Rosi, N. L.; Mirkin, C. A. Chem. Rev. 2005, 105, 15471562. (b) Rosi, N. L.; Giljohann, D. A.; Thaxton, C. S.; LyttonJean, A. K. R.; Han, M. S.; Mirkin, C. A. Science 2006, 312, 1027-1030. (c) Liu, J.; Cao, Z.; Lu, Y. Chem. Rev. 2009, 109, 1948-1998. (d) Katz, E.; Willner, I. Angew. Chem., Int. Ed. 2004, 43, 6042-6108. (e) Wang, H.; Yang, R. H.; Yang, L.; Tan, W. H. Acs Nano 2009, 3, 2451-2460. (f) Zhao, W.; Brook, M. A.; Li, Y. Chembiochem 2008, 9, 2363-2371. (g) Ricci, F.; Lai, R. Y.; Heeger, A. J.; Plaxco, K. W.; Sumner, J. J. Langmuir 2007, 23, 6827-6834. (h) Zhao, C.; Ren, J. S.; Qu, X. G. Chem. Eur. J. 2008, 14, 5435-5439.

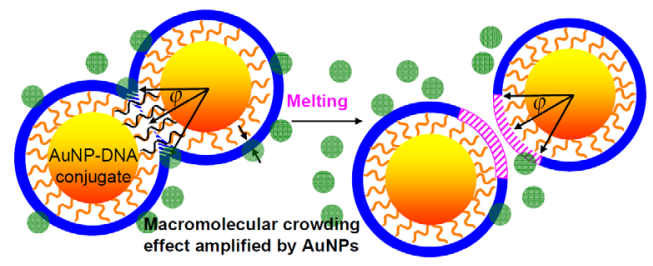

